\title{
Potentialities of Electrospun Polymeric Nanofibres in the Biomedical Field
}

\section{Maria Letizia Focarete ${ }^{1 *}$ and Chiara Gualandi ${ }^{2}$}

${ }^{1}$ Health Sciences and Technologies, Interdepartmental Center for Industrial Research (HST-ICIR), University of Bologna, Italy

${ }^{2}$ Department of Chemistry and National Consortium of Materials Science and Technology (INSTM, Bologna RU), University of Bologna, Italy

Electrospinning is a polymer processing technique that uses electrostatic forces to uniaxially stretch a viscoelastic jet derived from a polymer solution or melt, to produce continuous nanometric and micrometric fibres, typically assembled into non-woven mats [1]. It is an old technology and the first patent on the process was awarded in 1934 [2]. However, until the early 1990s only few publications dealt with this fabrication technique, and outside of the filtration industry there was little interest in electrospun nanofibres. It was only a couple of decades ago that the technique raised the interest of some research groups, first of all the Reneker's group at the University of Akron [3], that contributed to its spreading in research institutes all around the world. Since that time, the multi-potentialities of electrospinning started to be fully recognized and researchers gained understanding of the process governing this technology and acquired the capability to rule it. Electrospinning experienced then a rapid growth, and since that time over a hundred synthetic and natural polymers have been processed into electrospun fibres with diameters ranging from a few nanometers to micrometers. Further advancements in proper material design, in controlling process parameters, as well as the use of innovative spinning devices, allowed to dramatically expand the potentiality of the technology. Nanofibres made of materials different from polymers such as metal oxides, carbon, ceramics, as well as composite organic/ inorganic systems have been therefore obtained. The development of such systems made it possible to expand the list of possible uses of electrospun nanofibres as innovative functional products to be used in sensors, in catalytic and photo-catalytic systems and in energy storage systems (fuel cells, lithium ion batteries, solar cells).

The great potentiality of electrospun systems is mainly expressed in the biomedical field where they are predominantly applied for tissue engineering applications, drug delivery systems, diagnostics and biosensors, even if the possible uses in this field are practically unrestricted.

The high interest towards biomedical products fabricated through electrospinning principally arises from: (i) their inherent high surface to volume ratio; (ii) the possibility to engineer morphologically biomimetic scaffolds that resemble the fibrous structure of the natural extracellular matrix and (iii) pore interconnectivity that makes the entire porous structure fully accessible to chemical species. With respect to other nanofibre fabrication technologies (e.g. template synthesis, self-assembly, phase separation) electrospinning offers several advantages: (i) requires a very simple and cheap apparatus that can be scaled-up; (ii) a great variety of polymers, both synthetic and natural, can be processed into fibres; (iii) it enables the production of continuous fibres and the control of their dimension, surface texture, fibre orientation and mat porosity (iv) fibres of different materials and with different properties can be combined in the same mat; (v) fibre functionalization can be integrated during their production.

It turns out that an infinite possibility of materials, fibre dimensions, fibre orientations and fibre functionalization can be combined to engineer multi-functional hierarchically organized composites designed to fit the structural and chemical requirements of the specific biomedical application [4]. The first level of this hierarchically organization starts from the possibility to engineer the single fibre through blend, emulsion or coaxial electrospinning to obtain multi-component fibres loaded with a second polymer, or with nanoparticles, drugs or bioactive agents, thus providing an enormous versatility of material properties and functions. Moreover, nanofibre surface can be further functionalized with bioactive molecules and cell recognizable ligands throughout post-processing treatments such as plasma, wet chemical methods or graft polymerization $[5,6]$. The second level of complexity concerns the organization of nanofibres that can either be deposited into non-woven mat with random, aligned and ad-hoc orientation or that can be collected as bundles [7]. This allows controlling mat porosity, pore size and mat mechanical properties. The third level of electrospun system organization is related to the possibility of assembling different electrospun layers (or layers produced with other techniques such as rapid prototyping) to build a 3D structure with biochemical, structural and mechanical properties that can be tuned along the object dimensions.

We believe that electrospun products have the qualities to become the materials of the future in the biomedical field and that a revolutionary market will soon take place. Of course, only a synergic and effective collaboration between research institutes and companies will allow electrospinning to reach the deserved importance in the field. The following sections illustrate how the unique properties of non-woven nanofibrous structures are currently exploited to develop innovative biomedical devices.

\section{Tissue Engineering}

The scientific literature clearly demonstrated the powerful of using electrospinning over other methods of scaffold production thanks to its ease of use, scalability and, most of all, its intrinsic morphology that mimic the extracellular matrix of the native tissues. In addition, through the control over fibre orientation and dimension, combined with the addition of bioactive molecules (e.g. growth factors and peptides) and the possibility to construct multilayer structures, it is possible to fit cell proliferation, migration and differentiation to the desired tissue. The emerging market of companies such as Nicast Inc. (Israel), NanoFiber Solutions (USA) and Neotherix Ltd. (UK) attested the growing importance of these constructs in this field.

Notwithstanding the large number of publications dealing with the use of electrospun scaffolds in tissue engineering only a limited

*Corresponding author: Maria Letizia Focarete, Health Sciences and Technologies, Interdepartmental Center for Industrial Research (HST-ICIR), University of Bologna, Italy, E-mail: marialetizia.focarete@unibo.it

Received May 28, 2013; Accepted May 29, 2013; Published May 31, 2013

Citation: Focarete ML, Gualandi C (2013) Potentialities of Electrospun Polymeric Nanofibres in the Biomedical Field. J Tissue Sci Eng 4: e123. doi:10.4172/21577552.1000e123

Copyright: (c) 2013 Focarete ML, et al. This is an open-access article distributed under the terms of the Creative Commons Attribution License, which permits unrestricted use, distribution, and reproduction in any medium, provided the original author and source are credited. 
number of them explicitly recognize the intrinsic limitation of these materials with respect to cellular infiltration. Indeed, if the nanoscale dimension of the fibre is strongly desired to increase surface area and the biomimetic character of the scaffold, at the same time it induces the formation of small pores, thus hindering cell infiltration and making the electrospun mats behave as $2 \mathrm{D}$ substrates rather than $3 \mathrm{D}$ scaffolds. Addressing this critical drawback is essential to bring the promising research results to a higher level of feasibility. Many methods have been developed to increase the pore size of electrospun mats, maintaining at the same time the nanometric dimension of the fibres, thus obtaining 3D cell-scaffold constructs to enhance tissue integration [8]. However, the limited cellular in growth was demonstrated to be an advantage when dealing with barrier tissues (vascular graft, skin reconstruction, nerve repair, etc.). Here, electrospun scaffolds can display their maximum potentiality and will probably capture a major place in the near future.

\section{Wound Dressing}

The use of electrospun mats as wound dressing materials is gaining increasing popularity. The reason is obviously linked to the unique properties of electrospun membrane that perfectly fit with the functionalities required by an ideal wound dressing material [9]. First the highly porous structure and the complete pore interconnectivity are optimal for the drainage of the wound exudates and gaseous exchange. In addition, infections delaying healing can be prevented thanks to the small pores that inhibit external bacteria invasion as well as thanks to the ease addition of biocides and anti-inflammatory substances to the membrane during mat production. Another important aspect is the ability of electrospun membrane to fit the contour of the wound thanks to its high flexibility due to the fineness of its fibres. Moreover, the biomimetic feature of the membrane is believed to facilitate wound healing, thus limiting scar formation. Innovative commercial wound dressing materials produced by electrospinning are currently proposed by Neotherix Ltd. (UK) and Ri.Mos. Srl (Italy).

\section{Drug and Bioactive Agents Delivery}

Given the high surface-to-volume ratio and high porosity of electrospun fibres coupled with the easiness in encapsulating drugs within them, an intense research activity is dedicated to the use of electrospun mats as active substances delivery systems. Fibres have been initially loaded with the drug by blend electrospinning. However, this approach has intrinsic limitations since organic solvents typically used in electrospinning can have low compatibility with hydrophilic and charged molecules that might also lose or reduce their activity. Moreover, drug burst release from such a kind of fibres is often observed, due to the accumulation of the charged drugs on the surface of the fibres during the electrospinning process. Another approach consists in physically immobilizing the drugs on the fibre surface, exploiting nanofibrous constructs high surface area. In this case a fast drug release is achieved. Nowadays it is largely understood that the most promising approach for fabricating electrospun sustained release systems is coaxial electrospinning, where aqueous drug solution and polymer solution are maintained separated till the exit from the nozzles and the drug is placed in the core of the fibre protected by the polymeric shell. In this kind of system drug release occurs through diffusion across a barrier and burst release is avoided. Moreover, the possibility of using biodegradable polymers as drug reservoirs enables to control the drug release kinetics not only with a diffusion-driven mechanism but also with a degradation-driven one. The technology offers a wide versatility also in terms of encapsulated substances: antibiotics, proteins, growth factors, anticancer, antimicrobial, anti fungal and anti inflammatory agents have been successfully encapsulated in electrospun fibres.

\section{Anti-adhesion Barrier}

The adhesion between internal tissues and/or organs generally occurs after surgical procedures, such as abdominal-pelvic or gynaecological surgery, leading to severe clinical complications. In this context, the medical community is showing increasing interest towards the use of electrospun nanofibrous meshes as postsurgical anti-adhesion barrier. Indeed, electrospun meshes with tailored functionalization are good candidates for this specific application given their intrinsic ability to exert two main concomitant functions: (i) to physically separate the wound site from adjacent tissues and organs; (ii) to locally deliver therapeutic agents (antibiotics, anti-inflammatory agents, etc.). The barrier property of the meshes can be attributed to the limited cell migration within a properly designed scaffold, whereas the addition of antibiotics was demonstrated to help in completely preventing the adhesion [10]. A drug-loaded bioabsorbable electrospun barrier, beside reducing inflammation and preventing infection, can be degraded and absorbed once its function has been completed without the need of a further surgical procedure.

\section{Biosensors}

Besides being used as biomedical devices electrospun nanofibres are powerful tools to be applied in biosensors, although limited studies and technology transfer have been done up to now in this area. Biosensors are devices able to detect and quantify an analyte in a biological sample and to respond to biological or chemical stimuli. They are usually composed of a sensing element and a transducer that translates the interaction of the analyte (i.e. disease biomarkers, proteins, electrolytes, etc.) with the element into a measurable signal. This signal is used to quantify the concentration of the analyte which is usually associated with a specific medical condition. In several healthcare applications and in medical diagnostics there is the increasing need of more timely and reliable detection systems and research is rapidly moving towards ultrasensitive biosensors.

In this field, electrospun nanofibres are highly attractive products taking advantage of their remarkable specific surface area, high porosity and good transport properties that are ideal requirements for efficient molecule detection. Indeed, electrospun nanofibres with appropriate chemical and electrical functionality - can be used as the sensing element of biosensors for detection of biomolecules at very low concentration, since they can easily provide a huge number of sensing sites available for detection of the target analyte. In addition, electrospun nanofibres constitute efficient interfaces with the electronic measurement components of the biosensor, leading to rapid transduction and enhancement in signals, and they can be easily integrated with microelectrode interdigitated chips and microfluidic chambers to create lab-on-chip platforms. However, this application is still in its infancy and basic studies are still required to develop electrospun products with well-controlled orientation and size for achieving a proper and reproducible integration between electrospun nanofibres and useful devices [11].

\section{References}

1. Ramakrishna S, Fujihara K, Teo WE, Lim TC, Ma Z (2005) An introduction to electrospinning and nanofibers. World Scientific Publishing, Singapore.

2. Formhals A (1934) Process and apparatus for preparing artificial threads. US Patent No. 1975504A.

3. Doshi J, Reneker DH (1995) Electrospinning process and applications of electrospun fibers. J Electrostat 35: 151-160. 
Citation: Focarete ML, Gualandi C (2013) Potentialities of Electrospun Polymeric Nanofibres in the Biomedical Field. J Tissue Sci Eng 4: e123. doi:10.4172/2157-7552.1000e123

Page 3 of 3

4. TeoWE, Ramakrishna S (2009) Electrospun nanofibers as a platform for multifunctional, hierarchically organized nanocomposite. Compos Sci Technol 69: $1804-1817$

5. Yoo HS, Kim TG, Park TG (2009) Surface-functionalized electrospun nanofibers for tissue engineering and drug delivery. Adv Drug Deliv Rev 61: 1033-1042.

6. Gualandi C, Vo CD, Focarete ML, Scandola M, Pollicino A, et al. (2013) Advantages of surface-initiated ATRP (SI-ATRP) for the functionalization of electrospun materials. Macromol Rapid Commun 34: 51-56.

7. Teo WE, Ramakrishna S (2006) A review on electrospinning design and nanofibre assemblies. Nanotechnology 17: R89-R106.

8. Zhong S, Zhang Y, Lim CT (2012) Fabrication of large pores in electrospun nanofibrous scaffolds for cellular infiltration: a review. Tissue Eng Part B Rev 18: $77-87$

9. Zahedi P, Rezaeian I, Ranaei-Siadat SO, Jafari SH, Supaphol P (2010) A review on wound dressings with anemphasis on electrospun nanofibrous polymeric bandages. Polym Adv Technol 21: 77-95.

10. Zong X, Li S, Chen E, Garlick B, Kim KS, et al. (2004) Prevention of postsurgeryinduced abdominal adhesions by electrospun bioabsorbable nanofibrous poly(lactide-co-glycolide)-based membranes. Ann Surg 240: 910-915.

11. Ding B, Wang M, Wang X, Yu J, Sun G (2010) Electrospun nanomaterials for ultrasensitive sensors. Mater Today 13:16-27. 